\title{
MÁS ALLÁ DEL POSITIVISMO: UNA INTERPRETACIÓN LAKATOSIANA DE LA ENSENANZA DE LAS CIENCIAS
}

\author{
NÍAZ, M. \\ Departamento de Química, Universidad de Oriente. Apartado postal 90, Cumana. \\ Estado Sucre. Venezuela.
}

\section{SUMMARY}

The history of science shows that positivism was the dominant philosophy from about the end of the 19th century to the middle of the 20 th century. The main objective of this article is to present a critique of positivist ideas and show how the new philosophy of science, as represented by Lakatos, has important implications for science education.

\section{POSITIVISMO COMO UNA FILOSOFÍA DE LA CIENCIA A TRAVÉS DE LA HISTORIA}

La historia de la ciencia muestra que el positivismo era la filosofía predominante desde finales del siglo XIX hasta aproximadamente mediados del sigloxx (Wade 1977). El positivismo de A. Comte (1798-1857) sugiere que el mundo consiste en hechos positivos y reales y fenómenos observables, por lo tanto el conocimiento científico existe sólo en la descripción de estos fenómenos. $R$. Carnap (1891-1970), uno de los miembros mas destacados del positivismo lógico (perteneciente al Círculo de Viena), enfatiza el aspecto empírico (inductivista) de la ciencia. Según él, un término científico no tiene significación si no se refiere a un elemento de la experiencia. En otras palabras, términos científicos, tales como "electrón», «función de onda», $y$ «fuerza», no se pueden palpar a través de la experiencia y, por lo tanto, son problemáticos para los científicos positivistas, que enfatizan los hechos empíricos para poder observar, medir y manipular.

E. Mach (físico, 1838-1916) y W. Ostwald (fundador de físico-química, 1853-1932) fueron dos de los positivistas más prominentes que se opusieron a la teoría atómica en física y química. Ostwald, por ejemplo, hasta intentó derivar las leyes fundamentales (proporciones constantes y máltiples) sin la aytuda de la hipótesís atómica. Para los positivistas, como no se podía observar el átomo, se le consideraba en el mejor de los casos como una «ficción conveniente». Los positivistas tenian fobia contra cuaiquier cosa que no se podía observar. El trabajo de científicos eminentes como L. Boltzmann (1844-1906) y M. Planck (1858-1947) pudo contrarrestar la influencia de Mach y Ostwald para establecer la existencia de entidades que no se podían observar, tales como átomos, moléculas, electrones, cuantos y las partículas sub-atómicas.

\section{LA NUEVA FILOSOFÍA dE LA CIENCIA: MODELOS DE CAMBIO CIENTIFICO}

A partir de mediados de este sigio, el trabajo de los positivistas ha sido duramente criticado por autores como Hanson (1958), Popper (1959), Toulmin (1961), Kuhn (1962), Feyerabend (1970), Lakatos (1970) y Laudan (1977), lo que ha generado una nueva filosofía de la ciencia. De acuerdo con esta filosofía, nuestras observaciones dependen en parte de lo que ha sido la formación, experiencia y expectativas del observador; por lo tanto, las observaciones no pueden ser tan objetivas, sino que son impregnadas por el marco teórico del científico. Una de las grandes fallas del positivismo es la 
idea de que el conocimiento existe sin la interacción de un ser cognoscente. En otras palabras, el conocimiento existe mas allấ del científico, quien a través de sus investigaciones objetivas descubre las leyes, teorías y principios de nuestra realidad. La nueva filosofía de la ciencia presenta una perspectiva totalmente distinta. Lo que el científico observa e investiga es una «construcción» de la realidad de acuerdo con su formación, marco teórico y hasta valores sociales (Glasersfeld I989, Piaget 197 I, 1985, Piaget y García 1989). Hanson (1958) ha aclarado este problema, comparando la diferencia entre Jo observado por Kepler y Tycho al mirar el amanecer. Kepler considerabael sol como fijo y la tierra en movimiento. Tycho, al contrario, creía que la tierra estaba fija y el sol estaba en movimiento a su alrededor. Dado que los marcos teóricos de Kepler y Tycho eran diferentes, es obvio que los dos podrian haber observado el mismo objeto físico, pero la conceptualización o «construcción» de la realidad de cada uno era diferente. Lo más novedoso de la nueva filosofía de la ciencia es la preocupación por explicar el cambio en las teorías, mientras que anteriormente se pretendía, sobre todo, justificarlas.

\section{METODOLOGÍA DE LOS PROGRAMAS DE INVESTIGACION CIENTIFICA DE LAKATOS (MODELO DE CAMBIO CIENTIFICO)}

De más está decir que entre los protagonistas de la nueva filosofía de ia ciencia impera actualmente una ardua polémica (por ejemplo, Tiles 1992). La metodología de Lakatos $(1970,1971)$ consta de un centro firme, constituido por los aspectos esenciales de la teoría (heurística negativa) - que incluye sus supuestos fundamentales considerados irrefutables y un conjunto de hipótesis auxialiares (heurística positiva), compatibles con el centro firme. En contraste con la heurística negativa, la hipótesis en que se sustenta la heurística positiva es susceptible de cambio y de refutación. Los cientificos en general desarrollan sus teorías a través de la heurística positiva que permite modificaciones, lo que impide la refutación de la heurística negativa. Uno de los aspectos mas resaltantes de la metodología de los programas de investigación científica de Lakatos es que, en lugar de enfocar sobre las observaciones y falsaciones de las teorías, se enfatiza la competencia entre los programas de investigación 0 , en otras palabras, el cambio en el poder heurístico/explicativo de las teorías. El hecho de que haya evidencia empírica contra una teoría no significa que la teoría está falsada, siro que esto indica la necesidad de aumentar el poder heurístico/explicativo de la teoria, y eso sería factible cn la medida en que se desarrolle otra teoría en competencia (Lakatos 1970). La importancia de la filosofía de Lakatos, como un instrumento epistemológico para comprender la competencia entre los diferentes programas de investigación, ha sido reconocida en la enseñanza de las ciencias (Gilbert y Swift 1985, Níaz 1993a). Así mismo, López Rupérez (1990) ha enfatizado: «... Ia filosofía de la ciencia de l.akatos se puede convertir en un adecuado instrumento epistemológico de predicción/prescripción respecto de la evolución de la didáctica de las ciencias como disci- plina científica que ilumine el camino a recorrer por el investigador en particular y por la comunidad científica en general» (p. 72).

\section{UN EJEMPLO DE CAMBIO DE PROBLE- MA TICA: LA DIFERENCIA ENTRE SUJETO EPISTEMICO Y SUJETO PSICOLOGICO EN LA EPISTEMOLOGIA GENETICA DEPIAGET}

Piaget (1967) introdujo en la literatura de la psicología cognitiva una distinción epistemológica fundamental: la diferencia entre el sujeto epistémico (sujeto ideal que no existe) y el sujeto psicológico (sujeto real que se cncuentra en la vida cotidiana). Un psicólogo/educador, cuando observa que la gran mayoría de los sujetos no ałcanzan la etapa de razonamiento formal de Piaget (alrededor de los 15 años), siguiendo la filosofía del positivismo (empiricismo/inductivismo), concluye que Ia teoría de Piaget ha sido refutada. Esta interpretación de la teoría de Piaget ignora que en la epistemología genética de Piaget no se trata de individuos reales, sino que se trata de sujetos abstractos (epistémicos) que reflejan la racionalidad subyacente del hombre universal (Kitchener 1986). Esta metodología đe idealización (manipulación de las variables cognitivas) de Piaget se parece mucho a lo que hacen los científicos en las ciencias naturales para poder estudiar problemas complejos (ley de los gases ideales, leyes de Galileo y de Newton). Níaz (1991a), por ejemplo, ha demostrado una relación muy estrecha entre la metodología galileana y la construcción de la teoría de Piaget. Es importante señalar que, de acuerdo con Papineau (1976), el proceso de idealización forma parte de la heurística negativa dentro de lá filosofía lakatosiana, para ascgurar que la teoría no sea falsificada. La importancia para la enseñanza de las ciencias de la conceptualización de la diferencia entre el sujeto epistémico y psicológico ha sido demostrada por Níaz (1991b). Kitchener (1986) ha enfatizado que de acuerdo con la epistemología genética de Piaget, el sujeto epistémico construye el objeto epistémico (los conocimientos que nos rodean), y no puede haber el objeto epistémico sin un sujeto epistémico. La distinción entre el sujeto epistémico y picológico ha sido ignorada no sólo por los críticos de la obra de Piaget (Brainerd 1978 , Brown y Desforges 1977, Novak 1977), sino por aquellos que han utilizado el modelo piagetiano con frecuencia (por ejemplo, Lawson 1991). Más recientemente, Níaz (1992) ha demostrado que la transición epistémica entre el sujeto epistémico de Piaget y el metasujeto de Pascual-I eone se puede considerar como un cambio de problemática progresivo ("progressive problemshift») en el sentido lakatosiano.

\section{IMPLICACIONES PARA LA ENSEÑANZA DE LAS CIENCIAS}

En contraste con Popper y otros filósofos de la ciencia, una de las ideas más novedosas de Lakatos es que los hechos (datos empíricos) no pueden falsar una teoría, sino facititar la aparición de una nueva teoría con mayor poder heurístico/cxplicativo. De acuerdo con I akatos 
(1970), la historia de la ciencia muy pocas veces ha visto el dominio total de un programa de investigación; lo que más frecuentemente ocurre es una confrontación y competencia entre diferentes programas de investigación. Basándose en este aspecto de la metodología de Lakatos (1970), Níaz (1993b) ha demostrado cómo los diferentes programas de investigación en la enseñanza de las ciencias facilitan la transicion epistémica y no la falsación, es decir, el desarrollo de programas con mayor poder heurístico.

Es interesante observar como muchos de los textos de química general presentan una interpretación positivis* ta/inductivista de la revolución química durante el siglo xvifi, que se asocia con el nombre de A. Lavoisier (17431794). De acuerdo con esta interpretación, antes que Lavoisier hiciera sus experimentos, los científicos aceptaban la tcoría de G. StahI (1660-1734), quien sostuvo que todas las sustancias inflamables contienen un compo* nente llamado flogisto, y los materiales cuando se queman lo liberan hacia el aire. Así la teoría de flogisto sostenía que los metales al quemarse en el aire debían pesar menos, por la perdida de dicho componente. De acuerdocon la interpretación positivista, los experimentos de Lavoisier en el año 1772 demostraron que, cuando se quema cl azufre en el aire, su peso aumenta, lo que refutó definitivamente la teoría del flogisto. Muchos de los textos siguen esta interpretación; el siguiente es un ejemplo:

«Lavoisier (1743-1794) realizó experimentos que echaron por tierra la teoria del flogisto y dieron nacimiento a la química moderna» (Brown y Le May 1987, p. 5).

No obstante su popularidad, esta interpretación contradice los eventos históricos tal como ocurrieron. La historia de la química demuestra que aun en 1630 (mu- cho antes que Lavoisier) J. Rey (1630) habia reportado y era del conocimiento común que los metales al quemarse aumentan su peso. De acuerdo con Musgrave (1976), esto demuestra que siguiendo a los positivistas, si aceptamos el experimento de Lavoisier de 1772 como decisivo para la refutación de la teoría de flogisto, habría que concluir: «... la teoría de flogisto nacio refutada» (p. 183, cursiva original). Además muchos de los textos de química, al señalar la importancia de la teoría de oxígeno de Lavoisier, ignoran que su teoría sostenía equivocadamente que todos los ácidos contienen oxígeno.

Para comprender la diferencia entre la filosofía positivista/inductivista y lakatosiana, es importante comparar (Tabla I) las dos para la enseñanza de la estructura atómica. por ejemplo, Casi todos los textos resaltan la importancia, entre otros, de los modelos de Thomson, Rutherford y Bohr.

\section{COMPARACIÓN DE LA INTERPRETACIÓN POSITIVISTA Y LAKATOSIANA DE LOS MODELOS ATOMICOS}

Los historiadores positivistas, por ejemplo, resaltan el hecho de que cl trabajo de Bohr pudo explicar el caos que existía con respecto a los espectros de hidrógeno (series de Balmer y Paschen), antes de 1913. No obstante, de acuerdo con lakatos (1970), esta interpretación ignora el hecho de que Bohr ni siquiera había oído de las fórmulas de Balmer y Paschen antes de escribir la primera versión de su trabajo.

La diferencia más importante entre la conceptualización positivista/inductivista y lakatosiana es la idea de que la ciencia no se desarrolló en una línea recta, sino a través

Tabla I

Comparación de la interpretación positivista y lakatosiana de los modelos atómicos.

Interpretación

Modelo

Positivista

Lakatosiana

Lo importante es que hoy día los científicos aceptan que la relación carga/masa de las partículas en un tubo de rayos catódicos es una constante, independiente de los gases y del tubo.

Rutherford Indica la existencia de un núcleo con carga positiva y los electrones circundantes.

Bohr

Desartollóun modelo de átomo de hidrogeno que pudo explicar lo siguiente: las frecuencias emitidas en el espectro de hidrógeno coincidian con el trabajo experimental de Balmer y Paschen.
Lo impotantc es contrastar las diferentes teorías que luchaban para imponer su punto de vista. Es decir, la importancia del experimento consiste en la determinación de la relación carga/masa, para identificar las partículas como iones o partículas universales con carga.

Suministraba evidencia contra una teoría rival, es decir, el modelo atómico de Thomson, que postulaba el átomo como una esfera de densidad de masa y electricidad uniforme.

El objetivo fundamental de Bohr era de explicar la estabilidad paradójica del átomo de Rutherford -un modelo rival. 
de muchísimos puntos de vistas conflictivos. Es sólo a través de estos conflictos que generan la competencia entre programas rivales cómo la ciencia puede aumentar el poder explicativo/heurístico de sus teorías. Burbules y Linn (1991) han resumido esta idea en têrminos elocuentes: «Si la historia de la ciencia demuestra algo, es que a la larga todas las teorías más o menos son «equivocadas"» (p. 232).

\section{REFERENCIAS BIBLIOGRÁFICAS}

BRAINERD, C.J., 1978. The stage question in cognitive developmental theory, Behavioral and Brain Sciences, 2, pp. $173-213$

BROWN, G. y DESFORGES, C., 1977. Piagetian psychology and education: Time for revision, British Journal of Educational Psychology, 47, pp. 7-17.

BROWN, T.L. y LEMAY, H.E., 1987. Química: La ciencia

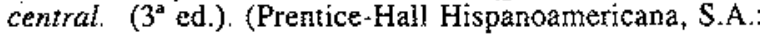
México, D.F.).

BURBULES, N.C. y LINN, M.C., 1991. Science education and philosophy of science: congruence or contradiction?, International Journal of Science Education, 13, pp. 227-24 I.

FEYERABEND, P.K., 1970. Against method: Outline of an anarchist theory of knowledge, en $M$. Radner y S. Winokur (eds.), Minnesota Studies in the Philosophy of Science (Vol. IV, pp. 17-130) University of Minnesota Press: Minncapolis).

GILBERT, J.K. y SWIFT, D.J., 1985. Towards a Lakatosian analy sis of the Piagetian and alternative conceptions research programs, Science Education, 69, pp. 681-696.

GIASERSFELD, E., von, 1989. Cognition, construction of knowledge, and teaching, Synthese, 80, pp. 121-140.

HANSON, N.R., 1958. Patterns of discovery. (Cambridge University Press: Cambridge).

KITCHENER, R.F., 1986. Piaget's theory of knowledge: Genetic epistemology and scientific reason. (Yale University Press: New Haven, CT).

KUHN, T.S., 1962. The structure of scientific revolutions. (University of Chicago Press: Chicago).

I.AKATOS, 1., 1970. Falsification and the methodology of scientific research programmes, en I. Lakatos y A. Musgrave (eds.), Criticism and the growth of knowledge, pp. 91-196. (Cambridge University Press: Cambridge). Trad. cast, 1983. La metodología de los programas de investigacion cientifica. (Alianza Editorial: Madrid, 1983).

LAKATOS, I., 1971. History of science and its rational reconstructions, en C.R. Buck y R.S. Cohen (eds.), Boston Studies in the Philosophy of Science, Vol. 28, pp. 91-135. (Reidel: Dordrecht, Holanda).

LAJUDAN, L., 1977. Progress and its problems. (University of California Press: Berkeley).

LAWSON, A.E., 1991. Is Piaget's epistemic subject dead?, Journal of Research in Science Teaching, 28, pp. 581-591.

I.OPFZZ RUPÉREZ, F, 1990. Epistemología y didáctica de las ciencias. Un análisis de segundo orden, Enseñanza de las Ciencias, 8, pp. 65-74.

MUSGRAVE, A., 1976. Why did oxygen supplant phlogiston? Research programmes in the Chemical Revolution, en C. Howson (ed.), Method and appraisal in the physical sciences.
The critical background to modern science, 1800-1905, pp. 181-209. (Cambridge University Press: Cambridge).

NÍAZ, M., 1991a. «From Galileo to Piaget: How do we construct epistemological theories?». Trabajo presentado en la 64 Conferencia Anual de la National Association for Research in Science Teaching (NARST), Lake Geneva, Wisconsin, Abril.

NÍAZ, M., 1991b. Role of the epistemic subject in Piaget's genetic epistemology and its importance for science education. Journal of Research in Science Teaching, 28, pp. 569-580.

NÍAZ, M., 1992. From Piaget's epistemic subject to PascualIeone's metasubject: Epistemic transition in the constructivistrationalist theory of cognitive development, International Journal of Psychology, 27, pp. 443-457.

NÍAZ, M., 1993a. Competing research programs in science education: A Lakatosian interpretation, Interchange, 24 (en imprenta).

NÍAZ, M., 1993b. Progressive "problemshifts» between different research programs in science education: A Lakatosian perspective, Journal of Research in Science Teaching, 30 (en imprenta).

NOVAK, J.D., 1977. An alternative to Piagetian psychology for science and mathematics education, Science Education, 61 , pp. 453-477.

PAPINEAU, D., 1976. Ideal types and empirical theories, British Journal for the Philosophy of Science, 27, pp. $137-146$

PIAGET, J., 1967. L' epistemologie et ses varietes, عn J. Piaget (ed.), Logique et connaissance scientifique, pp. 3-61. (Gallimard: Dordrecht)

PIAGET, I., 1971. Genetic epistemology. (Norton: Nueva York).

PIAGET, J., 1985. The equilibration of cognitive structures: The central problem of intellectual development. (Chicago University Press: Chicago).

PIAGET, J. y GARCIA, R., 1989. Psychogenesis and the history of science. (Columbia University Press: Nueva York).

POPPER, K., 1959. The logic of scientific discovery. (Hutchinson: Londres).

REY, J., 1630. Essays on an enquiry into the cause wherefore tin and lead increase in weight on calcination. Alembic Club Reprints, núm. 11, 1953.

TILES, J.E., 1992. Experimental evidence vs. experimental practice?, British Journal for the Philosophy of Science, 43, pp. 99-109.

TOULMIN, S., 1961. Foresight and understanding. (IN:Indiana University Press: Bloomington).

WADE, N., 1977. Thomas S. Kuhn: Revolutionary theorist of science, Science, pp. 143-145. 